\title{
DOES STAPHYLOCOCCUS SAPROPHYTICUS CAUSE ACUTE CYSTITIS ONLY IN YOUNG FEMALES, OR IS THERE MORE TO THE STORY? A ONE-YEAR COMPREHENSIVE STUDY DONE IN BUDAPEST, HUNGARY
}

\author{
JENNIFER AdEGHATE ${ }^{1}$, EMESE JUHÁSZ ${ }^{1}$, JÚliA PONGRÁCZ1, \\ ÉVA RimANÓCZY ${ }^{2}$, KATALIN KRISTÓF ${ }^{1 *}$ \\ ${ }^{1}$ Department of Laboratory Medicine, Semmelweis University, Budapest, Hungary. \\ ${ }^{2}$ Central Laboratory, Heim Pál Children's Hospital, Budapest, Hungary.
}

(Received: 18 December 2015; accepted: 15 January 2016)

Staphylococcus saprophyticus is a well-known urinary pathogen in acute cystitis in young females. We completed a retrospective overview of the distribution of urinary tract infections (UTIs) occurring in 2014, at Semmelweis University hospitals and at Heim Pál Children's Hospital. Six age-groups (ages 0-100) were examined, with the frequency of $S$. saprophyticus in females being: $0.1 \%(0-4), 0.7 \%$, (5-15), 7.4\% (16-24), 1.2\% (25-39), 0.4\% (40-59) and 0.1\% (60-100), and S. saprophyticus being the $3^{\text {rd }}$ most common pathogen in females aged 16-24. In males, S. saprophyticus was only isolated from those aged 5-15. Seasonal distribution of UTIs caused by $S$. saprophyticus showed that most infections occurred during the months of January, June, August and November. Antibiotic-resistance rates of amoxicillin, clindamycin, doxycycline, erythromycin, gentamicin and sulfamethoxazole-trimethoprim varied as follows: $0.9 \%, 32.7 \%, 19.6 \%, 34.6 \%, 0.9 \%$ and $0.9 \%$, respectively. Thirty randomly selected samples were analysed by pulsed-field gelelectrophoresis, and 28 different genotypes were identified. S. saprophyticus is involved in the pathogenesis of acute cystitis not only in young females, but also in other age-groups, and in young males as well. We did not find any significant seasonal occurrence in S. saprophyticus-caused UTIs. The infective strains were genetically diverse. Antibiotic-resistance does not pose any issue as of yet.

Keywords: Staphylococcus saprophyticus, UTI, acute cystitis

*Corresponding author; E-mail: kristof.katalin@med.semmelweis-univ.hu 


\section{Introduction}

Staphylococcus saprophyticus was first isolated from humans by Shaw et al. in 1951 [1], and its connection with urinary tract infections (UTIs), more specifically acute cystitis (otherwise known as "honeymoon cystitis"), was first published in 1962 by Torres Pereira [2]. In 1978, a Swedish study showed that $42.3 \%$ of UTIs in female patients aged 16-25 are caused by S. saprophyticus [3]. The importance of this microorganism as a causative agent in UTIs has been confirmed by multiple studies since then, and it has also been shown that its occurrence varies based on the population studied (i.e. age, gender, clinical features, seasonal distribution) [1, 4].

Over the years, we have obtained more and more information on the virulence and pathogenesis of $S$. saprophyticus. For one, it maintains its high affinity for urinary tract epithelium by producing multiple surface-associated proteins, such as haemagglutinin/adhesin, which is expressed in anaerobic environments, $S$. saprophyticus surface-associated fibrillar protein (Ssp), which mediates bacterial-urothelial cell-cell interactions, and SdrI, a newly discovered multifunctional fibronectin-binding protein, which is structurally similar to adhesion proteins found in Staphylococcus aureus and Staphylococcus epidermidis [5]. Other proteins include Aas (Autolysin adhesin), which is similar in function to the Atl autolysin found in $S$. aureus and $S$. epidermidis [6].

S. saprophyticus also produces an enzyme called 'urease', which breaks down toxic urea molecules in urine, allowing the bacteria to survive in the urinary tract. Other enzymes produced include: elastase, FAME (Fatty Acid Modifying Enzyme) and lipase (all are involved in the invasion of surrounding tissues). Diversification of these virulence factors allows $S$. saprophyticus to maintain its infectivity.

In our study, we conducted a retrospective analysis of patients presenting with UTIs between January and December of 2014 (one-year period) at hospitals affiliated with Semmelweis University, as well as at Heim Pál Children's Hospital, where the studied population comprised of paediatric patients.

Our aims were the following: (1) to determine the distribution of urinary tract pathogens, with a specific focus on the role of $S$. saprophyticus as a urinary tract pathogen (2) to clarify whether predisposition to $S$. saprophyticus infections is related to variables such as age and gender, (3) to determine whether there is any seasonal occurrence in $S$. saprophyticus infections, (4) to analyze antibiotic 
resistance profiles for $S$. saprophyticus strains isolated from the patients that were studied, and (5) to analyze the genetic diversity of these isolates. Thus, the main question we pose is the following: are UTIs truly more prevalent in young women as compared to the general population, or are they simply underrepresented in other patient-populations, such as young men and older females?

\section{Materials and Methods}

During the year of 2014, a total of 10,022 urinary tract pathogens were microbiologically confirmed from urine cultures of patients receiving treatment at hospitals associated with Semmelweis University and at Heim Pál Children's hospital in Budapest, Hungary. In our study, only one urine sample per patient was considered. Most urine cultures contained one pathogen, but we also took into consideration those that contained two (at most). Also, the samples were obtained in the presence of clinical signs and symptoms of infection. Isolates were identified by phenotypic methods and matrix-assisted laser desorption/ ionization time-of-flight mass spectrometry (MALDI-TOF) analysis. Time of collection of urine samples (month) and patient demographic data (age, gender) were collected. We separated the patients into six age-groups $(0-4,5-15,16-24$, 25-39, 40-59 and 60-100). We combined this data in order to show the distribution of age and gender in UTIs caused by different infective agents, as well as for the investigation of seasonal incidence in UTIs caused by S. saprophyticus and other common urinary pathogens. Antibiotic-resistance was tested in S. saprophyticus isolates using the disc diffusion method, and was interpreted according to the guidelines stated by the European Committee on Antimicrobial Susceptibility Testing (EUCAST) [7].

Thirty randomly selected $S$. saprophyticus isolates were stored at $-80{ }^{\circ} \mathrm{C}$ in $25 \%$ glycerol for subsequent molecular experimentation. Investigation of genetic diversity was executed using pulsed-field gel electrophoresis (PFGE) on the basis of an internal protocol modified for genotypic examination of coagulase-negative staphylococci, as described by Bradford et al. [8]. After enzymatic DNA-digestion of the thirty $S$. saprophyticus isolates using SmaI, the obtained DNA band patterns were analyzed. Determination of genetic relation between different strains was done according to the criteria set by Tenover et al. [9]. 


\section{Results}

\section{Distribution of urinary tract pathogens}

After reviewing the collected data, we illustrated the age and gender distribution of 10,022 UTI-causing pathogens (from 9,083 patients) during a one year period (2014), based on the criteria mentioned in the Materials and Methods section above (Table I). E. coli is the most common infective agent in females of all age-groups. Gram-negative bacteria belonging to the Enterobacteriaceae Family, as well as Enterococcus faecalis, were more frequently isolated in females aged 16+. A higher incidence of Pseudomonas aeruginosa-caused UTIs in females aged 0-4 is also noteworthy. S. saprophyticus is the third most commonly isolated urinary tract pathogen in females aged 16-24. The distribution is slightly more variable in males. E. coli is the most common urinary tract pathogen in the first two age-groups (0-4, 5-15), which resembles the distribution of the microorganism in females of the same age-groups. Subsequent age-groups, however, mainly acquired UTIs due to E. faecalis infections. UTIs in males caused by $S$. saprophyticus were found only in patients aged 5-15. Analyzing the age-distribution of UTIs in both males and females, it can be seen that UTIs are most common under the age of 5 and above the age of 40 (Fig. 1).

\section{Staphylococcus saprophyticus}

A total of 66 patients (61 female, 5 male) were shown to have S. saprophyticus as the causative pathogen in their UTI. S. saprophyticus was found in all female age-groups, though it mostly occurred in females aged 16-24, when the prevalence of UTIs, in general, is otherwise low. S. saprophyticus was also commonly isolated in females aged 25-39 (Fig. 1). In males, however, S. saprophyticus was only isolated from urine samples obtained from 5-15-year-olds.

Differences between the incidence of UTIs caused by S. saprophyticus and UTIs caused by other urinary tract pathogens show an interesting correlation in certain age-groups. The prevalence of UTIs is higher, in general, in both early childhood (ages $0-4$ ) and in the elderly (60-100). UTIs are overall less frequent in females aged 16-24 and 25-39, however, urinary tract infections caused specifically by $S$. saprophyticus are more common in these age-groups (Fig. 2).

In order to analyze seasonal changes in S. saprophyticus-caused UTIs, we compared the seasonal distribution of UTIs caused by S. saprophyticus to that of UTIs caused by all other urinary tract pathogens. Seasonal variability can be seen regarding the occurrence of UTIs, with most $S$. saprophyticus-caused UTIs 


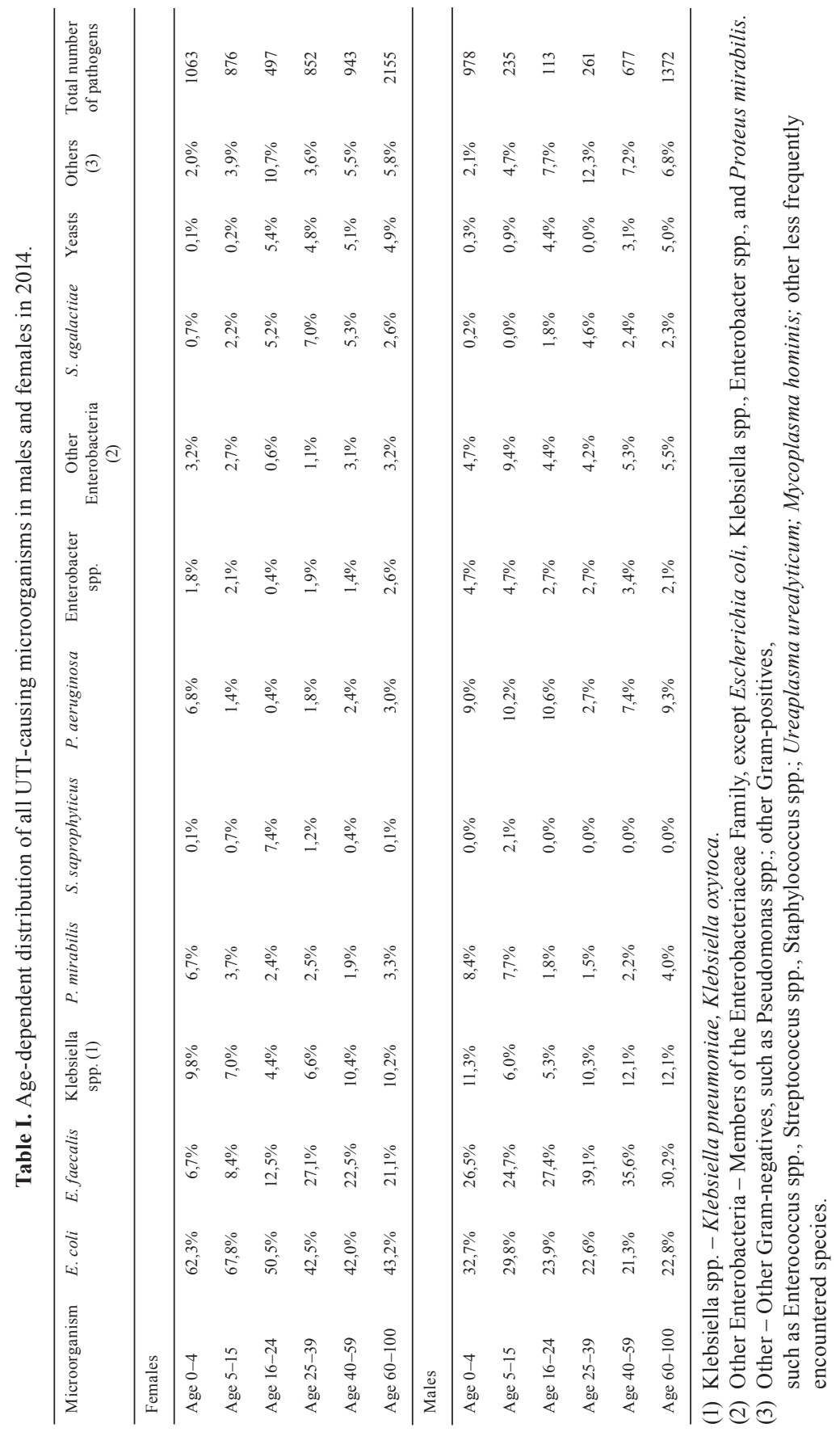




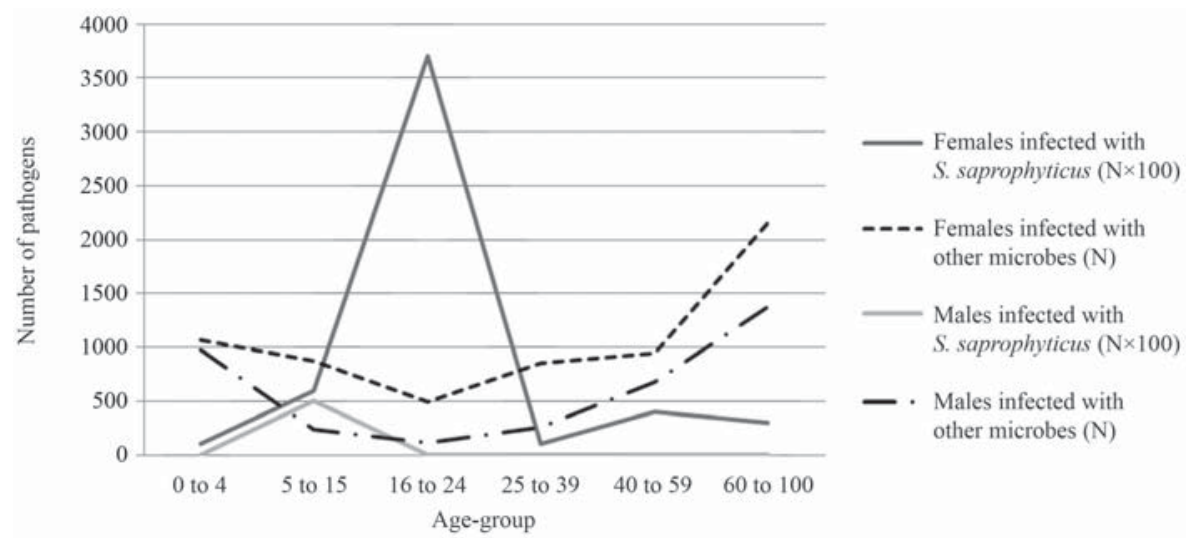

Figure 1. Age- and gender-dependent distribution of Staphylococcus saprophyticus-caused UTIs, compared to UTIs caused by other microorganisms.

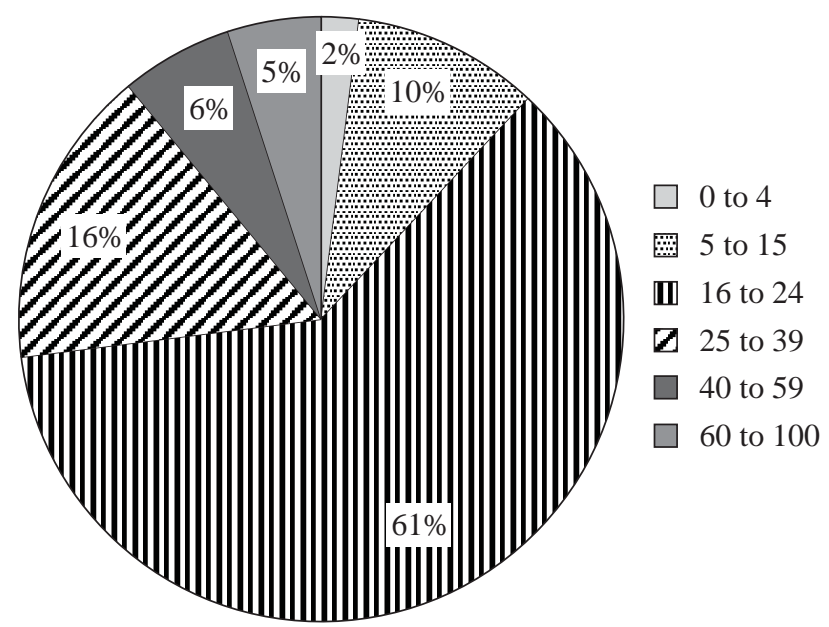

Figure 2. Age-dependency of Staphylococcus saprophyticus-caused UTIs in female patients $(\mathrm{n}=61)$.

occurring between mid-summer and mid-winter (larger peaks in occurrence can be seen in June, August, November and January) (Fig. 3).

Upon testing the antibiotic-resistance profile of $S$. saprophyticus, all isolates showed sensitivity to nitrofurantoin and fluoroquinolones, and with the exception of one, showed relatively high sensitivity to ampicillin as well (Fig. 4).

According to the criteria set by Tenover et al., we found 28 different $S$. saprophyticus genotypes based on the PFGE-analysis executed on 30 randomly selected samples [9]. 


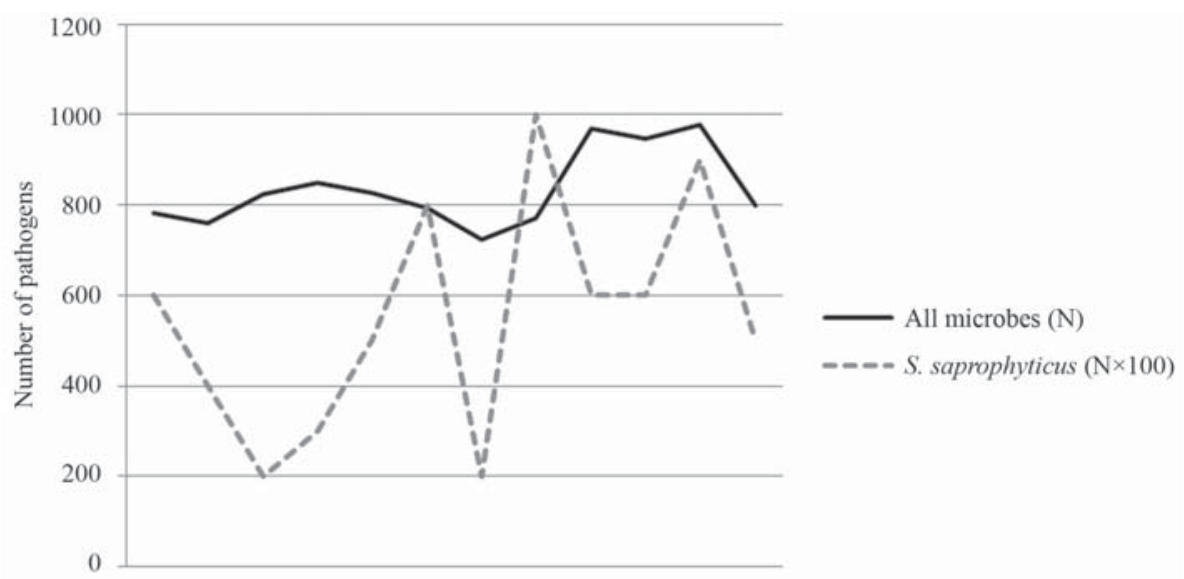

Jan Feb Mar Apr May Jun Jul Aug Sept Oct Nov Dec

Month

Figure 3. Seasonal distribution of all UTI-causing microorganisms in patients aged 0-100 in 2014

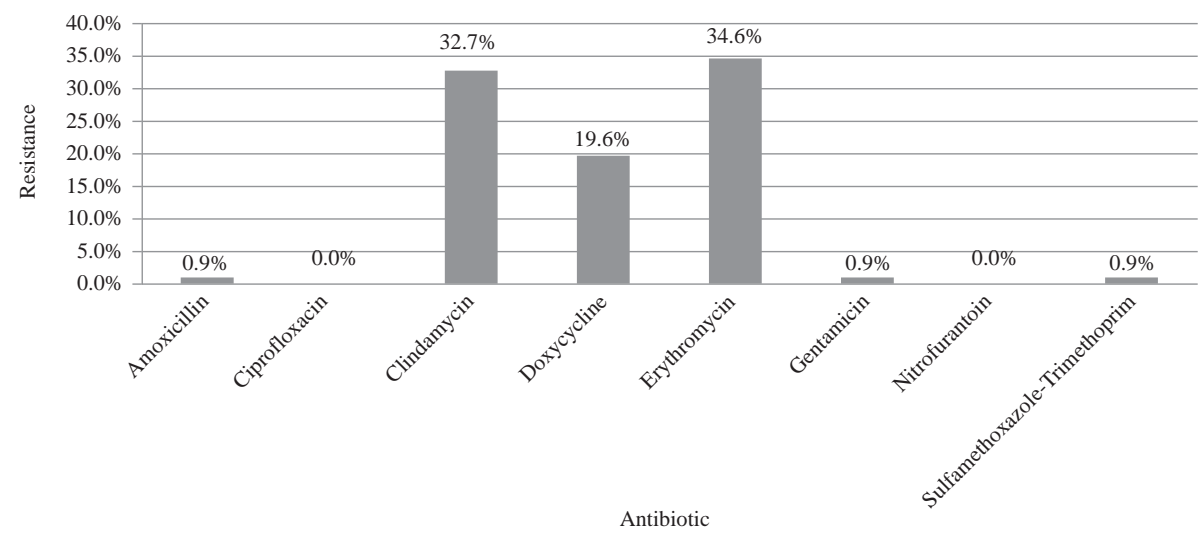

Figure 4. Antibiotic-resistance profile of Staphylococcus saprophyticus.

Resistance was tested with respect to Amoxicillin, Ciprofloxacin, Clindamycin, Doxycycline, Erythromycin, Gentamicin, Nitrofurantoin and Sulfamethoxazole-Trimethoprim

\section{Discussion}

Staphylococcus saprophyticus is a microbe well-known to be involved in the ocurrence of urinary tract infections (UTIs). Several previous studies have discussed its significance, especially in the development of acute cystitis in young females, and more is being discovered about its pathogenic properties, including 
resistance to antibiotics [10]. The data we collected within the tested period show that there is, in fact, a relation between the type of uropathogen, and the agegroup and gender which that uropathogen affects.

In healthy individuals, it colonizes the gastrointestinal tract, with the most common site being the rectum (40\%) [4]. It is also a part of the normal bacterial flora of the female genital tract and perineum [1, 11], with female urogenital tract colonization in healthy females at $6.9 \%$ [12]. In women, the vicinity of the vagina to the outer opening of the urinary tract (i.e. the urethra) makes the possibility of extra-genital colonization by this bacterium higher in females than in males [4]. Factors that increase susceptibility to infection in males include urinary tract obstruction and the presence of indwelling urinary catheters. The role of sexual activity is a strong risk factor in contracting the infection as well, as it increases the possibility of translocation of bacteria from the perineal region to the distal urethra [11]. Also, disruption of the normal flora of the female genital tract has been shown to increase the occurrence of UTIs caused by S. saprophyticus [4].

Other risk factors include sexual promiscuity, and bathing in public baths. Some infections have also been associated with specific geographical properties, as well as with the handling and consumption of certain meats [13]. The proposed pathomechanism of UTIs caused by S. saprophyticus is ascension of the bacteria proximally within the urinary tract, after which they colonize the urinary tract epithelium, causing cystitis and more severe UTIs, such as acute pyelonephritis and nephrolithiasis. In contrast to other UTI-causing organisms, such as E. coli and Proteus spp., which have been isolated from asymptomatic patients, S. saprophyticus infections are mostly accompanied by typical symptoms of upper and lower UTIs, such as dysuria, pollakisuria, hematuria, pyuria and back pain [11]. Septicaemia and endocarditis are rare sequellae of UTIs caused by $S$. saprophyticus, but are shown to have occurred [11].

$E$. coli is the most common infective agent in females of all age-groups, and in males aged $0-4$ and 5-15. Other common pathogens implicated in UTIs include E. faecalis and different Enterobacteriaceae species. P. aeruginosa was also frequently isolated, but mostly in females aged $0-4$, and in males aged 5-15 and 16-24.

With respect to $S$. saprophyticus, we have obtained similar results to those found by other research groups. S. saprophyticus is most commonly isolated from the urine of young and middle-aged, sexually active women with UTIs [11]. This can be seen in our study as well, in that it was most commonly isolated from urine samples of females aged 16-24 and 25-39. Also, as a uropathogen, it is known to be the second most common cause of urinary tract infections after Escherichia coli, as stated by Eriksson et al. [3], which is also evident in 
our results. Overall, we have found that UTIs are most common in early childhood (ages $0-4)$ and in the elderly (60-100), which suggests an association between immune system function and susceptibility to UTIs.

Though the possibility of a higher incidence of $S$. saprophyticus-caused UTIs in other age-groups and amongst males has been implied in the past, so far, we have not found any specific information regarding the prevalence of this bacterium in males aged $5-15$. This poses an interesting question as to why only this age-group is affected in males. In males around the age of 5, an anatomical explanation could be plausible, in which the proximity of the distal gastrointestinal tract to the genital tract may lead to an increased risk of ascending UTIs. Around the age of 15, however, the possibility of early sexual activity may be more likely, which also raises the question as to whether or not S. saprophyticus may be implicated in sexually transmitted infections (STIs) [14].

A higher occurrence of $S$. saprophyticus-caused UTIs can be seen in the months of June, August, November and January. The similarity in the seasonal occurrence of S. saprophyticus-caused UTIs to the seasonal distribution shown by STIs (late summer and fall), also questions whether or not $S$. saprophyticus infections may be implicated in STIs [15].

S. saprophyticus has so far proven to be sensitive to most antibiotics used in UTIs (namely amoxicillin, ciprofloxacin, clindamycin, doxycycline, erythromycin, gentamicin, nitrofurantoin and sulfamethoxazole-trimethoprim), and so far, shows no threat in becoming resistant to these antibiotics. Compared to national antibiotic-resistance statistics from 2014 [16], we have observed the following: (our own resistance results in "\%" / National Center for Epidemiology (OEK) results in "\%") amoxicillin: 0.9/34.1, ciprofloxacin: 0.0/0.8, clindamycin: 32.7/19.1, doxycycline: 19.6/17.6, erythromycin: $34.6 / 36.0$, gentamicin: 0.9/0.4, nitrofurantoin: 0.9/1.4 and sulfamethoxazole-trimethoprim: 0.9/2.5). It can be seen that there is no notable difference between our results and those obtained nationally, except in the case of amoxicillin, to which $S$. saprophyticus had shown a much lower resistance in our tests $(0.9 \%)$, than in those done by OEK (34.1\%). Also, resistance to clindamycin was almost twice the value $(32.7 \%)$ of that obtained by OEK (19.1\%). These differences could be explained by the likelihood that we had observed a much smaller population than was examined nationally.

Using molecular methods to test for variability between the genomes of the $S$. saprophyticus isolates, we found 28 different genotypes out of 30 tested specimens. Therefore, based on our study alone, it is not possible to distinguish any dominant genotype. 


\section{Conclusion}

In our study, we demonstrated that the occurrence of S. saprophyticus infections depends highly on the population studied, meaning that predisposing factors such as age, gender, clinical progression, and even seasonal changes, may influence the incidence of the infection.

S. saprophyticus is a urinary pathogen that is a significant cause of acute cystitis not only in young women, but also in other age and gender populations. S. saprophyticus isolates show highly variable genetic characteristics due to differing sources of infection. Fortunately, antibiotic-resistance has not yet posed an issue in the treatment of UTIs caused by S. saprophyticus, as most genetic variants have been shown to possess high sensitivity to most of the commonly-used antibiotics.

\section{Acknowledgements}

We would like to thank Natasa Pesti and Kinga Gothar for their invaluable work in the technical aspects of our study. We would also like to give special thanks to Professor Barna Vásárhelyi for providing the means to complete this project.

The Ethical Committee of Department of Laboratory Medicine Institute has reviewed and approved the use of laboratory and patient data for the purpose of this analysis.

\section{Conflict of Interest}

The authors declare that there is no conflict of interests regarding the publication of this paper.

\section{References}

1. Widerström, M., Wiström, J., Sjöstedt, A., Monsen, T.: Coagulase-negative staphylococci: update on the molecular epidemiology and clinical presentation, with a focus on Staphylococcus epidermidis and Staphylococcus saprophyticus. Eur J Clin Microbiol Infect Dis 31(1), 7-20 (2012).

2. Torres Pereira, A.: Coagulase-negative strains of staphylococcus possessing antigen 51 as agents of urinary infection. J Clin Pathol 15, 252-253 (1962). 
3. Eriksson, A., Giske, C., Ternhag, A.: The relative importance of Staphylococcus saprophyticus as a urinary tract pathogen: distribution of bacteria among urinary samples analysed during 1 year at a major Swedish laboratory. APMIS 121, 72-78 (2012).

4. Raz, R., Colodner, R., Kunin, C. M.: Who are you - Staphylococcus saprophyticus? CID 40, 896-898 (2005)

5. Sakinc, T., Kleine, B., Michalski, N., Kaase, M., Gatermann, S. G.: SdrI of Staphylococcus saprophyticus is a multifunctional protein: localization of the fibronectin-binding site. FEMS Microbiol Lett 301(1), 28-34 (2009).

6. Hell, W., Meyer, H. G., Gatermann, S. G.: Cloning of aas, a gene encoding a Staphylococcus saprophyticus surface protein with adhesive and autolytic properties. Mol Microbiol 29(3), 871-881 (1998).

7. EUCAST breakpoints: http://www.eucast.org/clinical_breakpoints/.

8. Bradford, R., Abdul Manan, R., Daley, A. J., Pearce, C., Ramalingam, A., D’Mello, D., Mueller, Y., Uahwatanasakul, W., Qu, Y., Grando, D., Garland, S., Deighton, M.: Coagulase-negative staphylococci in very-low-birth-weight infants: inability of genetic markers to distinguish invasive strains from blood culture contaminants. Eur J Clin Microbiol Infect Dis 25, 283-290 (2006).

9. Tenover, F. C., Arbeit, R. D., Goering, R. V., Mickelsen, P. A., Murray, B. E., Persing, D. H., Swaminathan, B.: Interpreting chromosomal DNA restriction patterns produced by pulsed-field gel electrophoresis: criteria for bacterial strain typing. J Clin Microbiol 33, 2233-2239 (1995).

10. Szasz, M., Lehotkai, N., Kristóf, K., Szabó, D., Nagy, K.: Prevalence and antimicrobial resistance of uropathogens in different inpatient wards. Acta Microbiol Immunol Hung 56(4), 375-387 (2009).

11. Levinson, W.: Review of Medical Microbiology and Immunology (2010). 11th ed.; pp. 94-99.

12. Rupp, M. E., Soper, D. E., Archer, G. L.: Colonization of the female genital tract with Staphylococcus saprophyticus. J Clin Microbiol 30, 2975-2979 (1992).

13. Hedman, P., Ringertz, O.: Urinary tract infections caused by Staphylococcus saprophyticus. A matched case control study. J Infect 23, 145-153 (1991).

14. Jordan, P. A., Iravani, A., Richard, G. A., Baer, H.: Urinary tract infection caused by Staphylococcus saprophyticus. J Infect Dis 142, 510-515 (1980).

15. Gatermann, S. G., Crossley, K. B.: Urinary tract infection. In: Crossley, K. B., Archer, G. (Eds). The staphylococci in human disease. New York: Churchill Livingston pp. 493-508 (1997).

16. National Centre of Epidemiology, Hungary: www.oek.hu. 